\title{
Generalized Divided Differences, with Applications to Generalized B-Splines
}

Guido Walz

Nr. 139

Februar 1992

PD Dr. Guido Walz

Lehrstuhl für Mathematik IV

Universität Mannheim

W-6800 Mannheim 1

Germany 


\title{
Generalized Divided Differences, with Applications to Generalized B-Splines
}

\author{
Guido Walz
}

\begin{abstract}
Within the theory of spline functions, there was always great interest in the study of B-splines, i.e. splines with a finite support. In addition to the classical polynomial case, there exist also various approaches to the definition of B-splines from other function classes, such as trigonometric or hyperbolic ones (cf. section 0 ). In the present paper we define B-splines from a rather general function space, which covers almost all existing approaches as special cases. The only condition that the spaces under consideration must satisfy is that of being translation invariant, a fundamental property which we are going to define in section 2 .

Our definition of generalized B-splines is based on generalized divided differences, which go back to Popoviciu [14] and were further investigated by Mühlbach [10,11]. In the first section of this paper we therefore study these operators and prove new results, such as a contour integral representation and a multistep-formula; the latter one expresses - in closed form - a generalized divided difference of order $m+j$ by those of order $m$, for arbitratry $j \in \mathbb{N}$. This makes it possible to compute generalized divided differences recursively, even if the underlying function space is spanned by a non-complete Chebyshev system.
\end{abstract}

\section{AMS Subject Classification: $\quad 41 \mathrm{~A} 15,65 \mathrm{D} 07$}

Key Words and Phrases: Generalized B-Splines, Divided Differences, Recursion Schemes. 


\section{Introduction}

Within the theory of spline functions, there was - starting with the fundamental work of Curry and Schoenberg [3, 4] - always great interest in such splines, which have a finite support, the so-called B-splines. There exist a lot of interesting studies in this field, and in addition to the Schoenberg/Curry paper we only refer here to de Boor [1]; for a nice compendium of the properties of these functions see e.g. Schumaker [16], Nürnberger [13] or Meinardus [9].

In the last decade, authors also began to study B-splines from non-polynomial spline spaces, such as trigonometric, hyperbolic or Chebychevian ones, see e.g. the papers of Schumaker $[15,17,18]$, Lyche and Winther [7] and Lyche [6]. The approach to these various types of B-splines is very similar in all cases, namely the use of a suitable divideddifference operator, applied to a proper generalization of what is called "truncated-powerfunction" in the polynomial case.

In the present paper we give a unified approach to almost all these different types of splines, i.e. we define - using generalized divided differences - a general type of Bspline function, which covers the ones listed above as special cases. The only condition that the spaces under consideration must satisfy is that of being translation invariant, a fundamental property which we are going to define later.

The organization of the paper is as follows: In section 1 we begin with the definition of generalized divided differences, which is originally due to Popoviciu [14] (cf. also Karlin [5] and Mühlbach [10,11]). Then we prove a "multistep formula" for generalized divided differences in closed form, i.e. a relation which allows us to compute a generalized divided difference of order, say, $m+j$, directly from those of order $m$ for arbitrary $j \in \mathbb{N}$. This generalizes results of Mühlbach $[10,11]$ and enables us to compute generalized divided differences recursively, even if the underlying function space is spanned by a non-complete Chebyshev system. Furthermore, we give a contour integral representation of the generalized divided difference operator.

In section 2 we define B-splines from a quite general function space. This is done by applying the divided difference operator from section 1 to a proper generalization of the well-known truncated power functions. Moreover, we apply our result from section 1 in 
order to obtain a representation of generalized B-splines via a complex contour integral; this covers known results for the case of polynomial (Meinardus [8]) and exponential splines (Walz [19]).

In the final section 3 we apply some of our results to an (hopefully) illustrative example, which is a little bit different from the standard cases of polynomial, trigonometric, hyperbolic or exponential splines. However, as we shall see, all these spline classes are covered by our general approach.

\section{Generalized Divided Differences}

The so-called generalized divided differences, introduced by Popoviciu [14], will be the basis for our investigations. We are going to use the following notation: For functions (resp. functionals) $f_{0}, \ldots, f_{k}$ and numbers (resp. functions) $y_{0}, \ldots, y_{k}$, we set

$$
\operatorname{det}\left(\begin{array}{cccc}
f_{0} & f_{1} & \cdots & f_{k} \\
y_{0} & \cdots & \cdots & y_{k}
\end{array}\right):=\operatorname{det}\left(\begin{array}{cccc}
f_{0}\left(y_{0}\right) & \cdots & \cdots & f_{0}\left(y_{k}\right) \\
\vdots & \vdots & \vdots & \vdots \\
\vdots & \vdots & \vdots & \vdots \\
f_{k}\left(y_{0}\right) & \cdots & \cdots & f_{k}\left(y_{k}\right)
\end{array}\right)
$$

Then the suitable definition of generalized divided differences is as follows:

Definition 1.1: Let $x_{\nu}, \ldots x_{\nu+k}$ be $k+1$ mutually distinct points from a real interval $I$, and let the real functions $\left\{f_{0}, \ldots, f_{k}\right\}$ form a (not necessarily complete) Chebyshev system on $I$.

Then the generalized divided difference $\Delta_{F}$ of a function $f$ with respect to the space

$$
F:=\operatorname{span}\left\{f_{0}, \ldots, f_{k}\right\}
$$

is defined as

$$
\Delta_{F}\left(x_{\nu}, \ldots, x_{\nu+k} ; f\right):=\frac{\operatorname{det}\left(\begin{array}{cccc}
f_{0} & \cdots & f_{k-1} & f \\
x_{\nu} & \cdots & \cdots & x_{\nu+k}
\end{array}\right)}{\operatorname{det}\left(\begin{array}{cccc}
f_{0} & \cdots & f_{k-1} & f_{k} \\
x_{\nu} & \cdots & \cdots & x_{\nu+k}
\end{array}\right)}
$$

We shall also use the notation $\Delta_{F}^{\nu, k}(f)$. The number $k$ is called the order of the divided difference. 
Obviously, $\Delta_{F}$ is a linear functional on $F$, which annihilates the subspace $F_{k-1}:=\operatorname{span}\left\{f_{0}, \ldots, f_{k-1}\right\}$ of $F$ and is normalized by

$$
\Delta_{F}^{\nu, k}\left(f_{k}\right)=1
$$

Therefore, some ideas developed in [2] can be applied, which will allow us to obtain some interesting new results on generalized divided differences.

The first one is the following "multistep formula", which contains certain known recurrence relations for generalized divided differences as special cases, cf. [10, Theorem 1], and [11, Corollary 2.16]. One should also compare Theorem 2.1 in [11] and Theorem 4.3 in [2].

Theorem 1.2: For $\nu \in \mathbb{Z}, m \in \mathbb{N}_{0}$ and $j \in \mathbb{N}$ with $m+j \leq k$ we have the identity

$$
\Delta_{F}^{\nu, m+j}(f)=\frac{\operatorname{det}\left(\begin{array}{cccc}
\Delta_{F}^{\nu, m} & \cdots & \cdots & \Delta_{F}^{\nu+j, m} \\
f_{m} & \cdots & f_{m+j-1} & f
\end{array}\right)}{\operatorname{det}\left(\begin{array}{cccc}
\Delta_{F}^{\nu, m} & \cdots & \cdots & \Delta_{F}^{\nu+j, m} \\
f_{m} & \cdots & f_{m+j-1} & f_{m+j}
\end{array}\right)},
$$

provided that the denominator in (1.2) is different from zero.

Proof. Let us try to write $\Delta_{F}^{\nu . m+j}$ as a linear combination of the $\Delta_{F}$ 's of order $m$, i.e. search for coefficients $a_{0}, \ldots, a_{j}$, such that

$$
\Delta_{r^{\prime}}^{\nu, m+j}(f)=\sum_{\mu=0}^{i} a_{\mu} \Delta_{r}^{\nu+\mu, m}(f) .
$$

Putting in (1.3) successively $f=f_{m}, \ldots, f_{m+j}$, we see that the $a_{\mu}$ 's are solutions of the following linear system of equations:

$$
\left(\begin{array}{cccc}
\Delta_{F}^{\nu, m}\left(f_{m+j}\right) & \Delta_{F}^{\nu+1, m}\left(f_{m+j}\right) & \cdots & \Delta_{F}^{\nu+j, m}\left(f_{m+j}\right) \\
\Delta_{F}^{\nu, m}\left(f_{m+j-1}\right) & \Delta_{F}^{\nu+1, m}\left(f_{m+j-1}\right) & \cdots & \Delta_{F}^{\nu+j, m}\left(f_{m+j-1}\right) \\
\vdots & \vdots & \vdots & \vdots \\
\Delta_{F}^{\nu, m}\left(f_{m}\right) & \Delta_{F}^{\nu+1, m}\left(f_{m}\right) & \cdots & \Delta_{F}^{\nu+j, m}\left(f_{m}\right)
\end{array}\right) \cdot\left(\begin{array}{c}
a_{0} \\
\vdots \\
\vdots \\
a_{j}
\end{array}\right)=\left(\begin{array}{c}
1 \\
0 \\
\vdots \\
0
\end{array}\right),
$$

and application of Cramer's rule proves the assertion of the theorem.

Obviously, formula (1.2) can be used in order to compute divided differences of order $m+j$ directly from those of order $m$, i.e. without using the expressions

$$
\Delta_{F}^{\nu, m+1}, \ldots, \Delta_{F}^{\nu, m+j-1} \text {. }
$$


This is of course essential if we have a non-complete Chebyshev system $F$, i.e. if some of the numbers in (1.4) might not be defined.

Let us investigate some special cases of Theorem 1.2. If $m=0$, then (1.2) is nothing else but a complicated form of the definition (1:1) (with $j$ instead of $k$ ), since $\Delta_{F}^{\nu, 0}(f)=f\left(x_{\nu}\right) / f_{0}\left(x_{\nu}\right)$.

Now let $m$ be arbitrary and $j=1$. Then we have

$$
\begin{aligned}
\Delta_{F}^{\nu, m+1}(f) & =\frac{\operatorname{det}\left(\begin{array}{cc}
\Delta_{F}^{\nu, m}(f) & \Delta_{F}^{\nu+1, m}(f) \\
\Delta_{F}^{\nu, m}\left(f_{m}\right) & \Delta_{F}^{\nu+1, m}\left(f_{m}\right)
\end{array}\right)}{\operatorname{det}\left(\begin{array}{cc}
\Delta_{F}^{\nu, m}\left(f_{m+1}\right) & \Delta_{F}^{\nu+1, m}\left(f_{m+1}\right) \\
\Delta_{F}^{\nu, m}\left(f_{m}\right) & \Delta_{F}^{\nu+1, m}\left(f_{m}\right)
\end{array}\right)} \\
& =\frac{\Delta_{F}^{\nu, m}(f)-\Delta_{F}^{\nu+1, m}(f)}{\Delta_{F}^{\nu, m}\left(f_{m+1}\right)-\Delta_{F}^{\nu+1, m}\left(f_{m+1}\right)},
\end{aligned}
$$

since $\Delta_{F}^{\nu, m}\left(f_{m}\right)=1$. This is precisely the "usual" recurrence formula for generalized divided differences, which is a very fundamental property and was found by Mühlbach in [10] with other methods.

Finally, analyzing the case $j=2$ in the same way would lead to a formula similar to the two-step-formula of Corollary 2.16 in [11].

It is well-known (see e.g. [12]) that the polynomial divided differences possess a representation via a complex contour integral. The following theorem gives the corresponding result for the generalized divided differences.

Theorem 1.3: For arbitrary, but fixed $\nu$ and $k$, and with the notations from above, let $A(z)$ denote some entire function, which has the simple zeros $x_{l^{\prime}}, \ldots, x_{\nu+k}$ and no others, and let $C$ be a simply closed and rectifiable curve in the complex plane, such that the points $x_{\nu}, \ldots, x_{\nu+k}$ lie inside of it. Furthermore, assume that the functions $f, f_{0}, \ldots, f_{k}$ have analytic continuations into the complex plane, and that for $j=0, \ldots, k-1$

$$
\frac{\left|f_{i}(z)\right|}{|A(z)|}=o\left(|z|^{-1}\right) \quad \text { for }|z| \rightarrow \infty
$$

Then, if the number

$$
\Omega:=\frac{1}{2 \pi i} \int_{C:} \frac{f_{k}(z)}{A(z)}
$$


is different from zero, the generalized divided difference $\Delta_{F}$ has the representations

$$
\Delta_{F}\left(x_{\nu}, \ldots, x_{\nu+k i} ; f\right)=\frac{1}{2 \pi i \Omega} \int_{C} \frac{f(z)}{A(z)} d z
$$

and

$$
\Delta_{F}\left(x_{\nu}, \ldots, x_{\nu+k} ; f\right)=\frac{1}{\Omega} \sum_{\mu=\nu}^{\nu+k} \frac{f\left(x_{\mu}\right)}{A^{\prime}\left(x_{\mu}\right)}
$$

The proof of (1.6) is similar to that of Theorem 3.3 in [2], to which we refer here, while (1.7) follows easily through the residue theorem.

The case of polynomial divided differences is recovered by setting $f_{i}(z):=z^{j}$, $j=0, \ldots, k$, and $A(z):=\left(z-z_{\nu}\right) \cdots\left(z-z_{\nu+k}\right)$. In this case we have $\Omega=1$.

\section{Applications to Generalized B-Splines}

In this section we shall use the generalized divided differences in order to define generalized B-splines, i.e. spline functions with minimal support, which belong piecewise to the space $F$. To do this, this space should possess a certain property, which we are now going to define. We shall always work on the whole real line; restrictions to a finite interval are easy to manage.

Definition 2.1: Let, for some $m \in \mathbb{N}, F$ denote the linear space

$$
F:=F_{m}:=\operatorname{span}\left\{f_{0}, \ldots, f_{m}\right\} \subset C^{m}(\mathbb{R}) \text {. }
$$

We say that this space has the translation property (or that it is translation invariant), if for each $f \in F$ and each fixed $x \in \mathbb{R}$ the mapping $f(t-x)$, regarded as a function of $t$, belongs to $F$; in other words, if for all $x, t \in \mathbb{R}$ and for all $f \in F$ there exist coefficients $c_{0}(x), \ldots, c_{m}(x)$, such that

$$
f(t-x)=\sum_{j=0}^{m} c_{j}(x) \cdot f_{j}(t) .
$$

Easy examples for spaces possessing the translation property are the space $\Pi_{m}$ of polynomials and the space

$$
E_{m}:=\operatorname{span}\{1, \exp (t), \ldots, \exp (m t)\}
$$


of real exponential sums. Furthermore, spaces of functions which satisfy an algebraic addition theorem, such as trigonometric or hyperbolic sums, possess the translation property. A counterexample is given by the two-dimensional space $\operatorname{span}\{1, \log (t)\}$.

From now on, we assume all spaces under consideration to possess the translation property. For the definition of generalized B-splines, which is our aim, we shall need a proper generalization of the truncated power-functions; this will be done in the next definition.

Definition 2.2: $\quad$ Let, for some $m \in \mathbb{N}, m \geq 2$, the spaces

$$
F_{m}:=\operatorname{span}\left\{f_{0}, \ldots, f_{m}\right\} \text { and } F_{m-1}:=\operatorname{span}\left\{f_{0}, \ldots, f_{m-1}\right\}
$$

possess the translation property. Furthermore, let there exist a function $h(t)=$ $h_{m-1}(t) \in F_{m-1}$, which is strictly positive or strictly negative on $\mathbb{R}^{+}$and has a zero of order $(m-1)$ in $t=0$.

We say that a function $g(t)=g_{m-1}(t)$ is a generalized truncated power function or a Green's function of $F_{m-1}$, if it can be written in the form

$$
g_{m-1}(t)= \begin{cases}0, & \text { if } t \leq 0 \\ h_{m-1}(t), & \text { if } t>0\end{cases}
$$

We note in passing that the function $g(t)$ belongs to the class $C^{m-2}(\mathbb{R})$. We now are in the position to prove the following fundamental theorem:

Theorem 2.3: $\quad$ With the notations and definitions from above, define the following function of $x$ :

$$
B_{m \nu}(x):=\Delta_{F_{m}}\left(x_{\nu}, \ldots, x_{\nu+m} ; g_{m-1}(t-x)\right)
$$

where the divided difference has to be taken with respect to $t$. Then the following assertions are valid:

1) The function $B_{m \nu}$ is a spline function from the space $S F_{m-1}(\mathbb{R})$, i.e. it is $(m-2)$-times continuously differentiable on $\mathbb{R}$ and its restriction to each knot interval $I_{k}:=\left[x_{k}, x_{k+1}\right], k=\nu, \ldots, \nu+m-1$, belongs to $F_{m-1}$;

2) The function $B_{m \nu}$ has finite support, i.e.:

$$
B_{m \nu}(x)=0 \quad \text { if } x \leq x_{\nu} \text { or } x \geq x_{\nu+m}
$$


Proof. Obviously (cf. also equation (1.3)) there exist coefficients $\alpha_{\nu}, \ldots, \alpha_{\nu+m}$, such that $B_{m \nu}$ can be written in the form

$$
B_{m \nu}(x)=\sum_{i=\nu}^{\nu+m} \alpha_{i} g\left(x_{i}-x\right) .
$$

Now let $x$ belong to the interval $I_{k}$. Then (2.2) becomes

$$
\sum_{i=k}^{\nu+m} \alpha_{i} h\left(x_{i}-x\right)
$$

which is a function from $F_{m-1}$, due to the translation invariance of this space.

Furthermore, (2.3) yields that the difference between the restrictions of $B_{m \nu}$ to $I_{h-1}$ and $I_{k}$ equals

$$
\alpha_{k} h\left(x_{k}-x\right) \text {, }
$$

and therefore has a zero of order $(m-1)$ in $x_{k} ;$ this proves the first assertion.

To show the second one, we first note that, if $x \geq x_{\nu+m}$, the argument of the divided difference in (2.1) is always zero, and therefore $B_{m \nu}(x)=0$ in this case.

Now let $x \leq x_{\nu}$. Then the translation invariance of $F_{m-1}$ yields the following equations:

$$
\begin{aligned}
B_{m \nu}(x) & =\Delta_{F_{m}}\left(x_{\nu}, \ldots, x_{\nu+m} ; h(t-x)\right) \\
& =\Delta_{F_{m}}\left(x_{\nu}, \ldots, x_{\nu+m} ; \sum_{j=0}^{m-1} c_{j}(x) f_{j}(t)\right) \\
& =\sum_{j=0}^{m-1} c_{j}(x) \Delta_{F_{m}}\left(x_{\nu}, \ldots, x_{\nu+m} ; f_{j}(t)\right) \\
& =0,
\end{aligned}
$$

since the generalized divided difference $\Delta_{F_{m}}$ annihilates the subspace $F_{m-1}$.

An application of Theorem 1.3 to (2.1) yields the following contour integral representation of the generalized B-spline $B_{n t \nu}$, which covers the corresponding results for polynomial [8] and exponential [19] B-splines as special cases.

Theorem 2.4: Adopt the notations and assumptions from Theorem 1.3, and let, for $x \in \mathbb{R}, C(x)$ denote some simply closed and rectifiable curve in the complex plane, such that all knots $x_{\mu}$ with $x<x_{\mu} \leq x_{\nu+m}$ and no others lie inside of $i t$.

Then the generalized B-spline function $B_{m \nu}$ has the representation

$$
B_{m \nu}(x)=\frac{1}{2 \pi i \Omega} \int_{C(x)} \frac{h_{m-1}(z-x)}{A(z)} d z
$$


Proof. Let $x \in I_{k}$, and denote the right hand side of (2.4) by $J_{m \nu}(x)$. Then the residue theorem yields

$$
\begin{aligned}
J_{m \nu}(x) & =\frac{1}{\Omega} \sum_{\substack{\mu=\nu \\
\mu>k}}^{\nu+m} \frac{h_{m-1}\left(x_{\mu}-x\right)}{A^{\prime}\left(x_{\mu}\right)} \\
& =\frac{1}{\Omega} \sum_{\mu=\nu}^{\nu+m} \frac{g_{m-1}\left(x_{\mu}-x\right)}{A^{\prime}\left(x_{\mu}\right)} . \\
& \stackrel{(1.7)}{=} \Delta_{F_{m}}\left(x_{\nu}, \ldots, x_{\nu+m} ; g_{m-1}(t-x)\right) .
\end{aligned}
$$

This completes the proof of Theorem 2.4.

We have seen so far how to define B-spline-functions for a rather arbitrary spline space $S F_{m-1}$. Of course one would like to compute the functions $B_{m \nu}$ via a recurrence relation, and this is possible thanks to the multistep fomula (1.2) (which contains the usual recursion formula (1.5) for generalized divided differences as special case). Moreover, using the relation (1.2) one can do this, even if the system $\left\{f_{0}, \ldots, f_{m}\right\}$ is a non-complete Chebyshev system; this is for example the case for trigonometric or hyperbolic splines.

\section{An Illustrative Example}

As pointed out above, our definition (2.1) covers most of the existing approaches to the various B-spline-functions, such as the polynomial, exponential, trigonometric and hyperbolic ones, cf. deBoor [1], Curry and Schoenberg [3, 4], Lyche [6], Lyche and Winther [7], Meinardus [8], Schumaker [15 - 18] and Walz [19]. We do not want to carry out now these special cases once again, but treat another example here in order to illustrate our approach.

Let us study the four-dimensional space $F_{3}$, which is spanned by the functions

$$
f_{0}(t):=1, f_{1}(t):=t, f_{2}(t)=\mathrm{e}^{t} \text { and } f_{3}(t):=t^{2}
$$

and its subspace

$$
F_{2}:=\operatorname{span}\left\{1, t, \mathrm{e}^{t}\right\}
$$

The function

$$
h_{2}(t):=1+t-\mathrm{e}^{t} \in F_{2}
$$


is strictly negative on $\mathbb{R}^{+}$and has a double zero in $t=0$; therefore, according to Definition 2.2, the function

$$
g(t)=g_{2}(t)= \begin{cases}0, & \text { if } t \leq 0 \\ 1+t-\mathrm{e}^{t}, & \text { if } t>0\end{cases}
$$

is a Green's function of $F_{2}$ and belongs to the class $C^{\mathrm{i}}(\mathbb{R})$. For given knot sequence $\left\{x_{j}\right\}$, we ask for B-splines from the space

$$
S F_{2}:=\left\{s \in C^{1}(\mathbb{R}) ; s(x)_{\mid I_{k}}=a_{k}+b_{k} x+c_{k} \mathrm{e}^{x}, k=\nu, \ldots, \nu+m-1\right\} .
$$

The solution to this is given Theorem 2.3; we have

$$
B_{3 \nu}(x)=\frac{\left(\begin{array}{cccc}
1 & 1 & 1 & 1 \\
x_{\nu} & x_{\nu+1} & x_{\nu+2} & x_{\nu+3} \\
\mathrm{e}^{x_{\nu}} & \mathrm{e}^{x_{\nu+1}} & \mathrm{e}^{x_{\nu+2}} & \mathrm{e}^{x_{\nu+3}} \\
g\left(x_{\nu}-x\right) & g\left(x_{\nu+1}-x\right) & g\left(x_{\nu+2}-x\right) & g\left(x_{\nu+3}-x\right)
\end{array}\right)}{\left(\begin{array}{cccc}
1 & 1 & 1 & 1 \\
x_{\nu} & x_{\nu+1} & x_{\nu+2} & x_{\nu+3} \\
\mathrm{e}^{x_{\nu}} & \mathrm{e}^{x_{\nu+1}} & \mathrm{e}^{x_{\nu+2}} & \mathrm{e}^{x_{\nu+3}} \\
x_{\nu}^{2} & x_{\nu+1}^{2} & x_{\nu+2}^{2} & x_{\nu+3}^{2}
\end{array}\right)}
$$

and an elementary calculation shows that indeed

$$
B_{3 \nu}(x)=0 \quad \text { if } x \leq x_{\iota} \text {, or } x \geq x_{\imath+3},
$$

according to Theorem 2.3 .

Furthermore, we calculate the coefficients $\alpha_{. i}$ in the representation

$$
B_{3 \nu^{\prime}}(x)=\sum_{j=\iota^{\prime}}^{\nu+3} \alpha_{j} g\left(x_{j}-x\right)
$$

This can be accomplished either, via the theorem of residues, applied to the contour integral representation (2.4), or via Cramer's rule, applied to (3.1). We choose the second possibility and obtain, since both numerator and denominator are "quasi-Vandermondians", easily the result: For $j=\nu, \ldots, \nu+3$, the coefficient $\alpha_{j}$ in (3.2) equals

$$
\alpha_{j}=\frac{N_{j}}{D}
$$

with

$$
N_{j}=\sum_{\substack{\mu=\nu \\ \mu \neq j}}^{\nu+3} \sigma_{\mu j} \cdot \mathrm{e}^{x_{\mu}} \cdot \prod_{\substack{u \leq n<\mu \leq j+3 \\ \alpha, \Delta \notin\{\mu, i\} .}}\left(x_{\beta}-x_{i r}\right), \text { where } \sigma_{\mu, j}= \begin{cases}(-1)^{\mu+j-1}, & \text { if } \mu<j \\ (-1)^{\mu+j}, & \text { if } \mu>j,\end{cases}
$$


and

$$
D=\sum_{\mu=\nu}^{\nu+3}(-1)^{\mu-\nu} \cdot \mathrm{e}^{x_{\mu}} \cdot \prod_{\substack{\nu \leq a<\beta \leq \nu+3 \\ \alpha, \beta \neq \mu}}\left(x_{\beta}-x_{\alpha}\right) .
$$

Finally, we would like to apply the multistep formula (1.2) to this example, i.e. to the B-spline $B_{3 \nu}(x)=\Delta_{F}^{\nu, \nu+3}\left(g_{2}(t-x)\right)$. In the notation of Theorem 1.2 we have here $m+j=3$, so we can take either $m=2, j=1$ or $m=1, j=2$ (in addition to the trivial cases $m=3$ and $m=0$ ).

Choosing $m=2, j=1$ leads to the representation

$$
B_{3 \nu}(x)=\frac{\left(\begin{array}{cc}
\Delta^{\nu \cdot 2}\left(\mathrm{e}^{t}\right) & \Delta^{\nu+1.2}\left(\mathrm{e}^{\ell}\right) \\
\Delta^{\nu: 2}(g(t-x)) & \Delta^{\nu+1: 2}(g(t-x))
\end{array}\right)}{\left(\begin{array}{ll}
\Delta^{\nu \cdot 2}\left(\mathrm{e}^{t}\right) & \Delta^{\nu+1.2}\left(\mathrm{e}^{t}\right) \\
\Delta^{\nu \cdot 2}\left(t^{2}\right) & \Delta^{\nu+1.2}\left(t^{2}\right)
\end{array}\right)},
$$

where each entry is a quotient of two $(3 \times 3)$-matrices. More suitable for numerical computations is the choice $m=1, j=2$, which leads to the formula

$$
B_{3 \nu}(x)=\frac{\left(\begin{array}{ccc}
1 & 1 & 1 \\
\Delta^{\nu .1}\left(\mathrm{e}^{\ell}\right) & \Delta^{\nu+1.1}\left(\mathrm{e}^{l}\right) & \Delta^{\nu+2.1}\left(\mathrm{e}^{l}\right) \\
\Delta^{\nu .1}(g(t-x)) & \Delta^{\nu+1.1}(g(t-x)) & \Delta^{\nu+2,1}(g(t-x))
\end{array}\right)}{\left(\begin{array}{ccc}
1 & 1 & 1 \\
\Delta^{\nu .1}\left(\mathrm{e}^{t}\right) & \Delta^{\nu+1,1}\left(\mathrm{e}^{t}\right) & \Delta^{\nu+2,1}\left(\mathrm{e}^{t}\right) \\
x_{\nu}+x_{\nu+1} & x_{\nu+1}+x_{\nu+2} & x_{\nu+2}+x_{\nu+3}
\end{array}\right)}
$$

where each entry is just a first order divided difference of the form

$$
\Delta^{j .1}(f)=\frac{f\left(x_{j}\right)-f\left(x_{. j+1}\right)}{x_{j}-x_{i+1}} .
$$

For the representation (3.3) we used the fact that, for all $j$,

$$
\Delta^{i .1}(t) \equiv 1 \text { and } \Delta^{i, 1}\left(t^{2}\right)=x_{j}+x_{j+1}
$$

In this paper, in particular in Theorem 2.3, we have seen how to define B-splinefunctions for a rather general spline space $S F_{m-1}$, and we worked out that the translation property seems to be fundamental for a space $F$ in order to allow the definition of B-splines via divided differences. We hope very much that this will inspire new investigations in the theory of non-polynomial spline functions. 


\section{References}

[1] C. deBoor: On Calculating with B-Splines.

J. Approx. Theory 6 (1972), 50 - 62

[2] C.Brezinski and G. Walz: Sequences of Transformations and

Triangular Recursion Schemes, with Applications in Numerical Analysis.

J. Comp. Appl. Math. 34 (1991), 361 - 383

[3] H. B. Curry and I. J. Schoenberg: On Spline Distributions and Their Limits: The Pólya Distribution Functions. Bull. Amer. Math. Soc. 53 (1947), 1114

[4] H.B. Curry and I.J. Schoenberg: On Pólya Frequency Functions IV:

The Fundamental Spline Functions and Their Limits.

J. Analyse Math. 17 (1966), 71 - 107

[5] S. Karlin: Total Positivity.

Stanford University Press 1968

[6] T. Lyche: A Recurrence Relation for Chebyshevian B-Splines.

Constr. Approx. 1 (1983), 155 - 173

[7] T. Lyche and R. Winther: A Stable Recurrence Relation for Trigonometric B-Splines. J. Approx. Theory 25 (1979), 266 - 279

[ 8] G. Meinardus: Bemerkungen zur Theorie der B-Splines.

In: Böhmer, Meinardus, Schempp (eds.): Spline-Funktionen.

Bibliographisches Institut, Mannheim/Zürich 1974, 165- 175

[9] G. Meinardus: Approximation Theory.

Springer, Berlin/Heidelberg, in preperation

[10] G. Mühlbach: A Recurrence Formula for Generalized Divided Differences and Some Applications. J. Approx. Theory 9 (1973), 165 - 172

[11] G. Mühlbach: The General Neville-Aitken-Algorithm and Some Applications. Numer. Math. 31 (1978), $97-110$

[12] N.E. Nörlund: Vorlesungen über Differenzenrechnung. Chelsea Publ., New York 1954

[13] G. Nürnberger: Approximation by Spline Functions. Springer, Berlin/Heidelberg/New York 1989

[14] T. Popoviciu: Sur le reste dans certaines formules linéaires d'approximation de l'analyse. Mathematica (Cluj) 1 (1959), 95 - 142

[15] L. L. Schumaker: On Tchebycheffian Spline Functions. J. Approx. Theory 18 (1976), $278-303$ 
[16] L. L. Schumaker: Spline Functions: Basic Theory. John Wiley and Sons, New York 1981

[17] L. L. Schumaker: On Recursions for Generalized Splines. J. Approx. Theory 36 (1982), $16-31$

[18] L. L. Schumaker: On Hyperbolic Splines. J. Approx. Theory 38 (1983), $144-166$

[19] G. Walz: Remarks on Exponential Splines, in Particular a Contour Integral Representation of Exponential B-Splines. Math. Meth. Appl. Sc. 11 (1989), $821-827$

PD Dr. Guido Walz

Lehrstuhl für Mathematik IV

Universität Mannheim

W-6800 Mannheim 1

Germany 